\title{
An experimental study measuring human annotator categorization agreement on commonsense sentences
}

\author{
Henrique Santos $^{1}$ (D), Mayank Kejriwal ${ }^{2}$ (D) Alice M. Mulvehill $^{1}$ (D), Gretchen Forbush ${ }^{1}$ (D) and \\ Deborah L. McGuinness ${ }^{1}$ \\ ${ }^{1}$ Tetherless World Constellation, Rensselaer Polytechnic Institute, NY, 12180, United States, and ${ }^{2}$ Information Sciences \\ Institute, University of Southern California, CA, 90292, United States \\ Corresponding author. E-mail: oliveh@rpi.edu
}

(Received 07 May 2021; Revised 28 May 2021; Accepted 28 May 2021)

\begin{abstract}
Developing agents capable of commonsense reasoning is an important goal in Artificial Intelligence (AI) research. Because commonsense is broadly defined, a computational theory that can formally categorize the various kinds of commonsense knowledge is critical for enabling fundamental research in this area. In a recent book, Gordon and Hobbs described such a categorization, argued to be reasonably complete. However, the theory's reliability has not been independently evaluated through human annotator judgments. This paper describes such an experimental study, whereby annotations were elicited across a subset of eight foundational categories proposed in the original Gordon-Hobbs theory. We avoid bias by eliciting annotations on 200 sentences from a commonsense benchmark dataset independently developed by an external organization. The results show that, while humans agree on relatively concrete categories like time and space, they disagree on more abstract concepts. The implications of these findings are briefly discussed.
\end{abstract}

Keywords: Commonsense reasoning; benchmarking; annotation; annotator agreement; commonsense theories

\section{Introduction}

Although there is no single, agreed-upon definition of commonsense, most definitions include the notion that commonsense is a shared human ability of understanding and judging everyday matters (Davis \& Marcus, 2015; Minsky, 2006). Commonsense reasoning in AI is about the development of computational agents that are capable of achieving human-like performance when presented with tasks that involve commonsense judgements (e.g. "True or False: If you throw a wine glass against a wooden floor, it will probably shatter", from the CycIC-entangled task). In recent years, significant computational progress has been achieved on this problem due to the advent of techniques such as transformer-based language representation models, of which Bidirectional Encoder Representations from Transformers (BERT) is an example (Devlin et al., 2019). Other examples include models such as Generative Pre-trained Transformer 3 (GPT-3) (Floridi \& Chiriatti, 2020), as well as recently released question-answering models such as UnifiedQA (Khashabi et al., 2020). Other enabling technologies include ensemble applications of symbolic and sub-symbolic AI approaches (Besold et al., 2017; Calegari et al., 2020; Cambria et al., 2020), which have also been successfully applied to difficult problems in AI, such as commonsense reasoning.

Because commonsense is broad, there is interest in both AI and cognitive science to better categorize the different kinds of commonsense reasoning that humans often rely on to navigate everyday life. While the problem of how humans categorize things in the world has been extensively studied (Mervis \& Rosch,

\footnotetext{
(c) The Author(s), 2021. Published by Cambridge University Press. This is an Open Access article, distributed under the terms of the Creative Commons Attribution licence (http://creativecommons.org/licenses/by/4.0), which permits unrestricted re-use, distribution and reproduction, provided the original article is properly cited.
} 
1981), recent literature has made considerable progress on the axiomatization of commonsense reasoning (Gordon \& Hobbs, 2017). Specifically, Gordon and Hobbs performed a comprehensive study of representational requirements for strategic planning. Planning strategies were collected in ten different domains, and formally represented. They found that 988 concepts could be considered common in all analyzed domains. These 988 concepts were then clustered into 48 representational areas. Eight of these areas (the ones selected for our experiment) were judged by the authors to be examples of foundational categories that are involved in human commonsense reasoning and are also commonly used in knowledge representation research. Our interest in these foundational categories is their potential to support the development of a formal logic for commonsense reasoning.

Our objective in this paper is to experimentally test the hypothesis of whether these 8 identified categories can be used by humans to reliably classify sentences into one or more of these categories. We test this hypothesis by designing and conducting an experiment where trained human annotators are tasked with independently classifying 200 commonsense sentences. To prove reliability, we robustly analyze the results to test for agreement among the annotators on their classifications. An annotationdriven experiment that, in structure, is similar to the one we propose is the work by Clinciu et al. (2021), where multiple annotators annotate papers published on commonsense reasoning, followed by a set of recommendations based on analysis. In contrast, we propose annotation of sentences from a commonsense reasoning benchmark into categories. Our end-goal is similar in that we draw on the results of this study to advocate for better design of commonsense benchmarks.

We note that such a reliable categorization, if it is found to exist, can facilitate several important purposes in the AI sub-community that investigates commonsense reasoning. First, it may help to design more comprehensive benchmarks (covering all the categories in the Gordon-Hobbs theory) for evaluating AI commonsense reasoners. Second, it may help to better understand the reasoners themselves, e.g., if one reasoner is doing better than another on some category (like time), but not some other category (like world states). In contrast, if the null hypothesis of human agreement underlying the hypothesis can be rejected with high significance, then it begs the question of whether the proposed axiomatization of commonsense is under-specified and needs further refinement. Our results show that, for some categories, this is indeed the case, while for others, there is agreement among human annotators.

\section{Methods}

Participants were asked to annotate a set of 200 sentences or 'prompts' that compose the CycICentangled (CycIC3) development dataset (described below), into one or more of the 8 categories (time, space, physical entities, classes and instances, sets, world states, and values and quantities) that have been identified by Gordon and Hobbs (2004) as relevant to most real-world commonsense reasoning tasks. Definitions for those categories are detailed, with references, in a recent book by the same authors (Gordon \& Hobbs, 2017). Cyc was originally proposed by Lenat and Guha (1989), and has been discussed in a number of publications over the decades (Lenat et al., 1990; 2010). Recently, Cyc released a benchmark dataset for use in evaluating natural-language-based AI agents on commonsense prompts that only require a True/False response. In its newest release, the CycIC3 development set (which is publicly available) contains 200 sentences that were designed to cover a reasonably representative set of commonsense concepts. Although CycIC3 provides some limited metadata about each of the sentences (including a category directly derived from the Cyc platform), the metadata is not directly aligned with a formal set of commonsense categories, since CycIC3's sentences were designed independently of the Gordon-Hobbs categories. Hence, these sentences/prompts provide a reasonable and independent corpus for acquiring annotations on those categories, without incurring bias in task construction.

\subsection{Annotators and annotation guidelines}

Five members actively working under the DARPA Machine Common Sense program participated as annotators in the experiment. While four out of the five annotators have computer science backgrounds, 
they have varying degrees of expertise. Prior to the experiment, the annotators received written instructions that included a brief description of each category, 2-5 example prompts per category, and annotation-scoring directions. The annotators were asked to provide a score, on a 5-point scale (with 1 being the least relevant, to 5 being the most relevant), for each of the 8 categories per prompt. Our goal in using this 5-point scale was to capture nuances in categorization that binary scores may have been incapable of capturing, and to ensure that annotators thought about the suitability of each category per prompt. Furthermore, by asking for a score for each category, rather than asking annotators to 'name' the most suitable category or categories per prompt, we mitigated the potential problem where annotators may have forgotten about some of the more difficult categories.

To elicit annotations, a spreadsheet was set up and shared on the Web with the five annotators. This spreadsheet contained the full set of 200 prompts, with a column for each of the 8 categories. The original spreadsheet, without annotations, is provided in the supplementary material.

In an effort to normalize the understanding of the categories, and to calibrate the scoring process with respect to the 5-point scale, the annotators were first instructed to independently annotate only the first 20 prompts. Once all participants completed this 'preliminary experiment', they met collectively to review and discuss the results. As a result of this discussion, the scale for scoring the relevance of each category was changed from 1-5 to $0-5$, where a score of 0 was used to indicate that a category is not relevant. Each participant was then instructed to complete, from the very beginning, the entire spreadsheet, containing the full set of 200 prompts, by scoring each prompt on a scale of $0-5$ for each of the 8 categories. Note that the 20 prompts used during the preliminary experiment were included in the final spreadsheet, although annotators were asked to fill in the entire spreadsheet from scratch. Postannotation comments were also solicited from all annotators to understand task difficulty.

\section{Metrics}

To quantify annotator agreement across the 8 categories, we used the balanced accuracy metric by treating, in turn, each annotator's categorization as the gold standard against which the other annotators' judgments would be evaluated. For binary judgments, balanced accuracy is the weighted proportion of correct answers (with respect to a given gold standard). In the supplementary material, we also provide the simple accuracy (unweighted proportion of correct answers). While a multi-class version of balanced accuracy is also available, we used the binary version of this metric to ensure robustness, since we wanted to ignore small scoring differences across annotators. We did this by using a threshold to convert the scaled annotations (on the $0-5$ point scale) across 8 categories into a binary judgment per category, for each prompt. For example, a threshold of 3 (that we use for our main results) indicates that a prompt scored as 0,1 , or 2 for some category by an annotator is not considered to belong to the category by that annotator (and hence is assigned a 'binary' label of 0 ), whereas prompts scored as 3, 4 or 5 are considered to belong to that category (binary label of 1 ). For each category, therefore, we can always recover a binary signal per prompt per annotator. We also consider the thresholds 1, 2, 4, and 5 and report additional results in the supplementary results. However, there is little qualitative difference when using these different thresholds, attesting to the robustness of our results.

We illustrate the process using an example. Suppose that the judgments of annotator A are treated as the gold standard (or 'ground-truth'). For a given category $\mathrm{C}$ (say, time) and threshold 3, we obtain a balanced accuracy for each of the other four annotators ( $, H, M$ and $R$ ) by first 'binarizing' their original scaled annotation and then computing a single, balanced accuracy metric per annotator for $\mathrm{C}$.

Since there are 8 categories, and 5 annotators, a total of $5 \times 4 \times 8=160$ balanced accuracy measurements are obtained and reported in Results. Assuming the null hypothesis that the binarized annotations of the ground-truth annotator and the annotator being evaluated are equally distributed, we can compute a p-value for each such pair of comparisons. For each category there are $5 \times 4 / 2=10$ comparisons, due to symmetry. Other works (Agresti, 1992) that have conducted similar categorization experiments to identify agreement or disagreement among subjects have used Cohen's Kappa statistic to compute 
Table 1. Balanced accuracy scores and p-value levels for each annotator pair for the Physical Entities (P.E.), Classes and Instances (C.I.), and Sets categories. A, G, H, M and R designate the five annotators.

\begin{tabular}{|c|c|c|c|c|c|c|c|c|c|c|c|c|c|c|c|}
\hline & \multicolumn{3}{|c|}{ A. } & \multicolumn{3}{|c|}{ G. } & \multicolumn{3}{|c|}{$\mathrm{H}}$. & \multicolumn{3}{|c|}{ M. } & \multicolumn{3}{|c|}{ R. } \\
\hline & P.E. & C.I. & Sets & P.E. & C.I. & Sets & P.E. & C.I. & Sets & P.E. & C.I. & Sets & P.E. & C.I. & Sets \\
\hline A. & & & & $0.50^{\star \star}$ & 0.55 & 0.60 & $0.63^{\star \star}$ & 0.56 & $0.58^{\star \star}$ & 0.54 & 0.47 & 0.45 & $0.57^{\star \star}$ & $0.52^{\star}$ & $0.55^{\star}$ \\
\hline G. & $0.50^{\star \star}$ & 0.55 & 0.61 & & & & 0.62 & 0.63 & $0.66^{\star \star}$ & $0.69^{\star \star}$ & $0.51^{\star}$ & 0.50 & 0.63 & 0.63 & 0.56 \\
\hline $\mathrm{H}$. & $0.61^{\star \star}$ & 0.56 & $0.56^{\star \star}$ & 0.62 & 0.63 & $0.62^{\star \star}$ & & & & $0.64^{\star \star}$ & 0.51 & $0.48^{\star \star}$ & 0.71 & $0.55^{\star}$ & $0.60^{\star *}$ \\
\hline M. & 0.54 & 0.47 & 0.44 & $0.70^{\star \star}$ & $0.51^{*}$ & 0.50 & $0.66^{\star \star}$ & 0.51 & $0.47^{\star \star}$ & & & & $0.62^{\star \star}$ & $0.50^{\star \star}$ & 0.51 \\
\hline R. & $0.57^{\star \star}$ & $0.51^{\star}$ & $0.56^{\star}$ & 0.63 & 0.62 & 0.56 & 0.74 & $0.55^{*}$ & $0.64^{\star \star}$ & $0.62^{\star *}$ & $0.50^{\star * *}$ & 0.51 & & & \\
\hline
\end{tabular}

Note: ${ }^{*} 0.01<p<=0.05{ }^{* *} p<=0.01$ 
Table 2. Balanced accuracy scores and p-value levels for each annotator pair for the World States (W.S.), and Values and Quantities (V.Q.) categories. A, G, H, M and R designate the five annotators.

\begin{tabular}{|c|c|c|c|c|c|c|c|c|c|c|}
\hline & \multicolumn{2}{|c|}{ A. } & \multicolumn{2}{|c|}{ G. } & \multicolumn{2}{|c|}{$\mathrm{H}$. } & \multicolumn{2}{|c|}{ M. } & \multicolumn{2}{|c|}{ R. } \\
\hline & W.S. & V.Q. & W.S. & V.Q. & W.S. & V.Q. & W.S. & V.Q & W.S. & V.Q. \\
\hline A. & & & 0.63 & 0.78 & $0.53^{\star \star}$ & 0.77 & $0.55^{\star *}$ & 0.61 & $0.53^{\star *}$ & $0.56^{\star}$ \\
\hline G. & 0.72 & 0.63 & & & $0.53^{\star \star}$ & 0.71 & $0.53^{\star *}$ & $0.55^{\star}$ & $0.55^{\star \star}$ & $0.53^{\star *}$ \\
\hline $\mathrm{H}$. & $0.67^{\star \star}$ & 0.67 & $0.58^{\star \star}$ & 0.79 & & & 0.64 & 0.62 & 0.55 & $0.59^{\star \star}$ \\
\hline M. & $0.73^{\star \star}$ & 0.65 & $0.59^{\star \star}$ & $0.66^{\star}$ & 0.64 & $0.75^{\star}$ & & & 0.55 & 0.61 \\
\hline R. & $0.64^{\star \star}$ & $0.63^{*}$ & $0.65^{\star *}$ & $0.64^{\star *}$ & 0.56 & $0.81^{\star *}$ & 0.55 & 0.67 & & \\
\hline
\end{tabular}

Note: ${ }^{\star} 0.01<p<=0.05^{\star \star} p<=0.01$

agreement and to check for chance agreement. Although we report the p-value scores in this paper, Cohen's Kappa statistic and the Kendall's $\tau$ scores are provided in the supplementary material.

\section{Results}

Our key experimental results are displayed in Tables 1, 2, and 3. In each table, the set of binarized annotations (using a threshold of 3) provided by the annotator in the first column is assumed to be the gold standard for that row, and all other annotators (listed in five major columns) are evaluated using the balanced accuracy metric described earlier. Since the balanced accuracy for an annotator (on any category) is trivially 1.0 when evaluated against itself, the cells falling along the diagonal are blank in each table.

Specifically, Table 1 reports results for the categories that are relatively general, i.e., Physical Entities (P.E), Classes and Instances (C.I), and Sets, and were used most often by the annotators (often in conjunction with other, less general categories). Compared to the categories in the other tables, there was considerable disagreement among annotators on the three categories in Table 1. With only two exceptions, balanced accuracy is below 70\%, even though two sets of commonsense, human judgments are being compared. For most results, the $\mathrm{p}$-value is well below 0.01 (indicated with $\mathrm{a}^{* *}$ ), meaning that the null hypothesis of annotator agreement can be rejected with high significance.

In complete contrast with Table 1 , there is much more agreement among the annotators on the more deterministic categories in Table 3, such as Time (Ti.), Space (Sp.), and Events (Ev.). For instance, there are very few balanced accuracies that are below $70 \%$ in Table 3 , and although the null hypothesis of agreement can be rejected for some pairs of annotators (for a category), there is weak or no significance for many others.

Results in Table 2 fall between the extremes in Tables 1 and 3. We find that balanced accuracies for categories that are considered less broad are higher, on average, than for those reported in Table 1. The two categories in this table, which include World States (W.S.) and Values and Quantities (V.Q.), have some degree of non-determinism, but are not as narrowly defined as the categories in Table 3.

During our data analysis, we varied the threshold to test if it leads to more agreement. The data for this additional analysis is included in the supplementary material. Results were largely consistent with those shown in Tables 1,2, and 3, attesting to the robustness of the experiment and its conclusions.

\section{Discussion}

While our main results suggest that the more deterministic subset (Time, Space, and Events) of the 8 categories identified by Gordon and Hobbs (2004) can potentially be used to categorize sentences in 
Table 3. Balanced accuracy scores and p-value levels for each annotator pair for the Time (Ti.), Space (Sp.), and Events (Ev.) categories. A, G, H, M and R designate the five annotators.

\begin{tabular}{|c|c|c|c|c|c|c|c|c|c|c|c|c|c|c|c|}
\hline & \multicolumn{3}{|c|}{ A. } & \multicolumn{3}{|c|}{ G. } & \multicolumn{3}{|c|}{$\mathrm{H}$. } & \multicolumn{3}{|c|}{ M. } & \multicolumn{3}{|c|}{ R. } \\
\hline & Ti. & Sp. & Ev. & Ti. & Sp. & Ev. & Ti. & Sp. & Ev. & Ti. & Sp. & Ev. & Ti. & Sp. & Ev. \\
\hline A. & & & & 0.71 & $0.66^{\star *}$ & $0.74^{\star \star}$ & 0.78 & 0.72 & 0.73 & 0.72 & $0.65^{\star}$ & 0.75 & $0.65^{\star \star}$ & $0.64^{\star \star}$ & 0.70 \\
\hline G. & 0.86 & $0.77^{\star \star}$ & $0.65^{\star \star}$ & & & & 0.87 & $0.80^{*}$ & $0.67^{\star \star}$ & 0.82 & 0.80 & $0.70^{\star \star}$ & 0.77 & 0.79 & $0.73^{\star *}$ \\
\hline $\mathrm{H}$. & 0.87 & 0.75 & 0.73 & 0.79 & $0.70^{\star}$ & $0.77^{\star \star}$ & & & & 0.80 & 0.70 & 0.75 & $0.71^{\star \star}$ & $0.69^{\star \star}$ & 0.84 \\
\hline M. & 0.76 & $0.74^{\star}$ & 0.74 & 0.73 & 0.77 & $0.81^{\star \star}$ & 0.77 & 0.78 & 0.74 & & & & $0.70^{\star \star}$ & $0.74^{\star}$ & 0.78 \\
\hline R. & $0.86^{\star *}$ & $0.78^{\star \star}$ & 0.69 & 0.89 & 0.84 & $0.83^{\star \star}$ & $0.89^{\star \star}$ & $0.84^{\star \star}$ & 0.82 & $0.91^{\star \star}$ & $0.81^{\star}$ & 0.77 & & & \\
\hline
\end{tabular}

Note: ${ }^{*} 0.01<p<=0.05 *{ }^{* *} p<=0.01$ 
commonsense benchmarks, the same results also show that there is much more annotator disagreement among the most general categories, such as Classes and Instances, and Sets.

To understand the difference more qualitatively, it is useful to consider two examples. For the first example, consider the prompt "If a chicken lays an egg, then the chicken existed before the egg". In our experiment, Time and Events were the categories that were consistently used to classify this prompt (using any threshold) by all of the annotators, indicating high agreement. Alternatively, the sentence prompt "Stabbing someone typically expresses love" could not reach agreement among all annotators for threshold $>=3$. Only the Events category was used by all annotators for this prompt, and only when considering threshold $<3$. While this prompt was also categorized into other categories such as Sets or Classes and Instances, annotators were not consistent among themselves. While reasons for this lack of consistency are not completely evident, for the second prompt, the annotators reported difficulty in classifying sentences about emotions and human preferences into any of the 8 presented categories. Some annotators also commented that they did not find much difference between some categories (e.g., Sets and Classes and Instances), implying that these categories may have been used interchangeably.

Sentence structure and language usage do not seem to be predictive of disagreement. Disagreement on categorization is also not correlated with difficulty in answering the prompt itself. For example, Cyc has claimed a human performance baseline of $100 \%$ for the CycIC3 benchmark, meaning that humans were able to answer each prompt correctly (true or false), despite significant disagreement in categorizing the prompts.

Earlier we noted that the Gordon-Hobbs theory contains 48 categories. We focused on the 8 that were identified as foundational. An important question that we hope to address in future work is whether the methodology presented in this paper can be successfully replicated on those categories. While a similar annotation exercise can be designed, some experimental caveats need to be borne in mind. First, as evidenced by annotator-feedback, annotators can sometimes get confused between categories and what they mean, even with eight categories. The problem is likely to get much worse if all 48 categories are presented to the annotators at the same time. Second, choice paralysis is a real issue that cannot be neglected when too many choices are presented to annotators. Other aspects are also important, such as the order in which the choices are presented. Cognitive fatigue may lead to less meaningful scores as annotators provide scores per sentence on increasing numbers of categories. Finally, at least some of the other 40 categories may fall in the 'abstract' or broad categories on which significant disagreement was observed even with 8 categories. Therefore, more annotators may be required to obtain statistically valid scores on those categories. While laying out an experimental study for all 48 categories is beyond the scope of this work, we believe that even formulating such a design would be a valuable area for future research.

Our long-term goal is to determine if a set of categories could reliably be used within a metadata layer to analyze the content of commonsense benchmarks. Potentially, a text classification algorithm might be able to automatically generate characterization for prompts in those benchmarks, as initially explored in Santos et al. (2021). The development of such an automated method will leverage the results discussed in this paper, and other results produced by researchers involved in the DARPA Machine Common Sense program. However, before such a classifier can be developed, methods for reducing human disagreement need to be devised. For example, annotation guidelines for the 8 categories may need to be better enumerated in future such exercises, and additional categories may be necessary for reducing ambiguity.

Acknowledgments. The authors thank the DARPA Machine Common Sense Program for supporting this research. We also acknowledge and thank Minor Gordon for conducting preliminary experiments leading up to the primary experiments reported herein, and Rebecca Cowan for contributing to the definitions of the identified categories.

Supplementary Materials. To view supplementary material for this article, please visit http://dx.doi.org/10.1017/exp.2021.9.

Author Contributions. Mayank Kejriwal and Henrique Santos conceived and designed the experiment. Alice M. Mulvehill and Henrique Santos established the methodology. Gretchen Forbush and Henrique Santos performed the data analysis. Henrique Santos, Alice M. Mulvehill, and Gretchen Forbush wrote the article. Mayank Kejriwal and Deborah L. McGuinness co-led the team effort and co-authored the article. 
Funding Information. This work is funded through the DARPA MCS program under award number N660011924033.

Data Availability Statement. The CycIC-entangled (CycIC3) dataset is available at https://github.com/steeter-cyclist/ CycIC3. The code used to compute scores is available at https://github.com/tetherless-world/mcs-formalization.

Conflict of Interest. None to declare.

\section{References}

Agresti, A. (1992). Modelling patterns of agreement and disagreement. In Statistical Methods in Medical Research (Vol. 1, pp. 201-218).

Besold, T. R., Garcez, A. D., Bader, S., Bowman, H., Domingos, P., Hitzler, P., Kühnberger, K.-U., Lamb, L. C., Lowd, D., Lima, P. M. V., de Penning, L., Pinkas, G., Poon, H., \& Zaverucha, G. (2017). Neural-symbolic learning and reasoning: A survey and interpretation. arXiv preprint arXiv:1711.03902.

Calegari, R., Ciatto, G., Denti, E., \& Omicini, A. (2020). Logic-based technologies for intelligent systems: State of the art and perspectives. Information, 11, 167.

Cambria, E., Li, Y., Xing, F. Z., Poria, S., \& Kwok, K. (2020). Senticnet 6: Ensemble application of symbolic and subsymbolic ai for sentiment analysis. Proceedings of the 29th ACM International Conference on Information \& Knowledge Management, pp. 105-114.

Clinciu, M.-A., Gkatzia, D., \& Mahamood, S. (2021). It's commonsense, isn't it? demystifying human evaluations in commonsense-enhanced nlg systems. Proceedings of the Workshop on Human Evaluation of NLP Systems (HumEval), pp. 1-12.

Davis, E., \& Marcus, G. (2015). Commonsense reasoning and commonsense knowledge in artificial intelligence. Communications of the ACM, 58, 92-103.

Devlin, J., Chang, M.-W., Lee, K., \& Toutanova, K. (2019). BERT: Pre-training of Deep Bidirectional Transformers for Language Understanding. Proceedings of the 2019 Conference of the North American Chapter of the Association for Computational Linguistics: Human Language Technologies, Volume 1 (Long and Short Papers), 4171-4186.

Floridi, L., \& Chiriatti, M. (2020). Gpt-3: Its nature, scope, limits, and consequences. Minds and Machines, 30, 681-694.

Gordon, A. S., \& Hobbs, J. R. (2004). Formalizations of commonsense psychology. AI Magazine, 25, 49-49. Number: 4.

Gordon, A. S., \& Hobbs, J. R. (2017). A Formal Theory of Commonsense Psychology: How People Think People Think. Cambridge University Press.

Khashabi, D., Min, S., Khot, T., Sabharwal, A., Tafjord, O., Clark, P., \& Hajishirzi, H. (2020). UNIFIEDQA: Crossing Format Boundaries with a Single QA System. Findings of the Association for Computational Linguistics: EMNLP 2020, 1896-1907.

Lenat, D., Witbrock, M., Baxter, D., Blackstone, E., Deaton, C., Schneider, D., Scott, J., \& Shepard, B. (2010). Harnessing Cyc to answer clinical researchers' ad hoc queries. AI Magazine, 31, 13-32. Number: 3.

Lenat, D. B., \& Guha, R. V. (1989). Building Large Knowledge-Based Systems; Representation and Inference in the Cyc Project (1st ed.). Addison-Wesley Longman Publishing Co., Inc.

Lenat, D. B., Guha, R. V., Pittman, K., Pratt, D., \& Shepherd, M. (1990). Cyc: Toward programs with common sense. Communications of the ACM, 33, 30-49.

Mervis, C. B., \& Rosch, E. (1981). Categorization of natural objects. Annual Review of Psychology, 32, 89-115.

Minsky, M. L. (2006). The Emotion Machine: Commensense Thinking, Artificial Intelligence, and the Future of the Human Mind. Simon \& Schuster. Google-Books-ID: AT0UIQEACAAJ.

Santos, H., Gordon, M., Liang, Z., Forbush, G., \& McGuinness, D. L. (2021). Exploring and Analyzing Machine Commonsense Benchmarks. Proceedings of the Workshop on Common Sense Knowledge Graphs.

Cite this article: Santos H, Kejriwal M, Mulvehill AM, Forbush G, McGuinness DL (2021). An experimental study measuring human annotator categorization agreement on commonsense sentences Experimental Results, 2, e19, 1-11. https://doi.org/10.1017/exp.2021.9 


\title{
Peer Reviews
}

\author{
Reviewing editor: Dr. Adín Ramírez Rivera
}

UNICAMP, Institute of Computing, Av. Albert Einstein 1251, Campinas, São Paulo, Brazil, 13083-872

This article has been accepted because it is deemed to be scientifically sound, has the correct controls, has appropriate methodology and is statistically valid, and has been sent for additional statistical evaluation and met required revisions.

doi:10.1017/exp.2021.9.pr1

\section{Review 1: An experimental study measuring human annotator categorization agreement on commonsense sentences}

Reviewer: Dr. Rafal Rzepka

Hokkaido University, Sapporo, Japan, 060-0808

Date of review: 18 May 2021

(C) The Author(s), 2021. Published by Cambridge University Press. This is an Open Access article, distributed under the terms of the Creative Commons Attribution licence (http://creativecommons.org/licenses/by/4.0), which permits unrestricted re-use, distribution and reproduction, provided the original article is properly cited.

Conflict of interest statement. Reviewer declares none

Comments to the Author: The paper presents empirical results showing agreement of human annotations for 8 of 48 representational areas of commonsense concepts proposed by Gordon and Hobbs. This line of work is important for the field of artificial intelligence because the manually created categories can fit what people treat as "common" but sometimes the proposed categorization is not as universal as its creators assume. However, there are several issues with the research and its presentation:

[evaluation depth] Common sense evaluation is problematic (see e.g. Clinciu et al. latest paper "It's Common Sense, isn't it? Demystifying Human Evaluations in Commonsense-enhanced NLG systems"); references about similar experiments might be missing.

[evaluation method] No explanation why the classic Kendall's $\tau$, Kolmogorov-Smirnov's D, or Cohen's Kappa (free-marginal kappa?) couldn't be used

[annotators info] Only five annotators with almost identical background (no information about sex or gender, probable problems with representativeness)

[data origin] CycIC dataset test set only has 3,000 sentences, where the 200 questions were taken from? No reference, probable problem with reproducibility.

[data choice] Why CycIC or widely used ConceptNet categories couldn't be used instead or compared?

[probable overstatement] The authors have chosen 8 areas of common sense and claim that their work can be helpful to evaluate remaining 40 (but as Gordon and Hobbs note, "the difference between these areas is in the degrees of interdependency that theories in these two groups require - these first eight representational areas can be developed in isolation from each other, whereas the latter forty cannot". 
Presentation

Is the data presented in the most useful manner? (40\%) 3/5

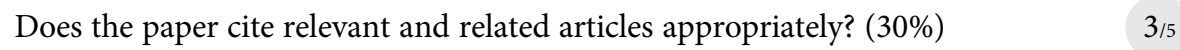

Context

3.5

Does the title suitably represent the article? (25\%)

Does the abstract correctly embody the content of the article? (25\%)

Does the introduction give appropriate context? (25\%)

Is the objective of the experiment clearly defined? $(25 \%)$

Analysis

Are the limitations of the experiment as well as the contributions of the experiment clearly outlined? $(20 \%)$ 


\section{Review 2: An experimental study measuring human annotator categorization agreement on commonsense sentences}

Reviewer: Prof. Erik Cambria (D)

Nanyang Technological University, Singapore, Singapore, 639798

Date of review: 22 May 2021

(c) The Author(s), 2021. Published by Cambridge University Press. This is an Open Access article, distributed under the terms of the Creative Commons Attribution licence (http://creativecommons.org/licenses/by/4.0), which permits unrestricted re-use, distribution and reproduction, provided the original article is properly cited.

Conflict of interest statement. Reviewer declares none

Comments to the Author: The manuscript is centered on a very interesting and timely topic, which is also quite relevant to EXPR themes. Organization of the paper is good and the proposed method is quite novel. The length of the manuscript is about right and presentation is good.

The manuscript, however, does not link well with relevant literature on commonsense computing, e.g., check latest trends on transformer models for commonsense validation. Also, recent works on the ensemble application of symbolic and subsymbolic AI for commonsense reasoning are missing.

Finally, add some examples of those 200 sentence for better readability and understanding of the paper. In fact, some EXPR reader may not be aware of the importance of commonsense. To this end, I also suggest to include some applications of commonsense computing, e.g., dialogue systems with commonsense and fuzzy commonsense reasoning for multimodal sentiment analysis.

\section{Score Card}

Presentation

4.0

Is the article written in clear and proper English? (30\%)

Is the data presented in the most useful manner? (40\%)

Does the paper cite relevant and related articles appropriately? (30\%)

Context

4.0

Does the title suitably represent the article? (25\%)

Does the abstract correctly embody the content of the article? (25\%)

Does the introduction give appropriate context? (25\%)

Is the objective of the experiment clearly defined? (25\%)

Analysis

Does the discussion adequately interpret the results presented? (40\%)

Is the conclusion consistent with the results and discussion? (40\%)

Are the limitations of the experiment as well as the contributions of the experiment clearly outlined? $(20 \%)$ 